\title{
Taking care of systemic sclerosis patients during COVID-19 pandemic: rethink the clinical activity
}

\author{
Antonina Minniti $^{1} \cdot$ Wanda Maglione $^{1} \cdot$ Francesca Pignataro $^{1} \cdot$ Carmela Cappadona $^{1} \cdot$ Roberto Caporali $^{1,2}$. $^{2}$ \\ Nicoletta Del Papa ${ }^{1,3}$ (10
}

Received: 7 May 2020 / Revised: 7 May 2020 / Accepted: 19 May 2020 / Published online: 27 May 2020

(C) International League of Associations for Rheumatology (ILAR) 2020

\begin{abstract}
COVID-19 outbreak has quickly spread worldwide, causing a high pressure on the health-care system. In Italy, from March 8, 2020, all the deferrable clinical activities have been suspended to increase the health care offer for COVID-19 patients. The hospital organization has been modified also in order to assure non-COVID-19 patients assistance. The Scleroderma Unit of ASST Pini-CTO Hospital, in Milan, in the region mostly hit by SARS-CoV-2 in Italy, follows more than 600 patients affected by systemic sclerosis (SSc). Patients with SSc need a close follow-up with a regular screening of organ involvement and frequent intravenous treatments. All SSc patients have been educated about ministerial directives to limit COVID-19 spread. The organization of our Scleroderma Unit has been quickly rethought to assure SSc patients assistance in safety for them and for healthcare workers during urgent visits or infusion therapies. Using electronic way of communication with frequent virtual contact and guarantying home deliveries of some therapies, we allowed a continuity of care also outside the Hospital.
\end{abstract}

Keywords COVID-19 $\cdot$ Patient care $\cdot$ Public health $\cdot$ Systemic sclerosis

\section{Introduction}

In December 2019, in the Hubei province of China, an epidemic began sustained by a coronavirus named severe acute respiratory syndrome-coronavirus-2 (SARS-CoV-2). The syndrome has been named coronavirus disease 2019 (COVID-19) and is characterized mainly by respiratory and systemic symptoms (cough, rhinorrhea, fever, arthralgia, myalgia, fatigue) [1]. The virus is transmitted during close contact through respiratory droplets and by fomites. It rapidly spread in the world, leading the World Health Organization officially to declare a pandemic state on March 11, 2020.

The virus diffusion is favored by asymptomatic subjects or patients with mild symptoms and by the long-time viral load

Nicoletta Del Papa

nicoletta.delpapa@asst-pini-cto.it

1 Department of Rheumatology, ASST Pini-CTO, Milan, Italy

2 Department of Clinical Sciences and Community Health and Research Center for Pediatric and Adult Rheumatic Diseases (RECAP.RD), University of Milan, Milan, Italy

3 Scleroderma Unit, Department of Rheumatology, ASST Pini-CTO, Milan, Italy disappearance (14 days or maybe longer). COVID-19 seems to have a lower mortality compared with other more important human coronavirus syndromes (SARS-1 and MERS), but a higher spread capability [1]. However, the disease could evolve to a severe interstitial pneumonia requiring mechanical ventilation; therefore, it is important to contain COVID-19 diffusion to avoid intensive care unit (ICU) saturation.

In Italy, the first case of secondary transmission was reported on February 18, 2020 in Codogno (Lombardy, North Italy). Since then, the virus spread in North regions and then quickly in the entire Country, leading to 107,709 confirmed cases and 24,648 deaths (http://www.salute.gov.it/portale/ nuovocoronavirus/dettaglioContenutiNuovoCoronavirus.jsp? lingua $=$ italiano $\& \mathrm{id}=5351 \&$ area $=$ nuovoCoronavirus $\& \mathrm{menu}=$ vuoto, last access April 22, 2020).

In order to contain the transmission, the Italian government disposed a lockdown from March 8, 2020 in North Italy and from March 11, 2020 in the rest of the country (still ongoing during the writing of this paper). The lockdown consists in the closing of all unnecessary activities and in the prohibition of all the people to move between different cities and municipalities, except for proven working needs and health reasons.

In Lombardy, the Italian region most hit from the epidemic, the regional government suspended from March 8, 2020 all 
the deferrable and non-urgent clinical activities, with the purpose to reduce inflow of in- and out-patients and to increase the health care offer for COVID-19 patients, in terms of capacity of beds and number of hospital staff. All the health-care system was needed to be rethought in order to guarantee also non-COVID-19 patients assistance, especially for patients with chronic diseases and requiring hospital treatment.

\section{Scleroderma Unit of ASST Pini-CTO Hospital}

The Department of Rheumatology of ASST Pini-CTO Hospital in Milan includes some specialized Units such as Early Arthritis Clinic, Lupus Clinic, Bone Unit, Sjögren Clinic, and Scleroderma Unit.

The Scleroderma Unit follows more than 600 patients suffering from systemic sclerosis (SSc). SSc is a rare systemic disease characterized by inflammatory, vascular, and fibrotic processes resulting in skin fibrosis and multiple organ manifestations, such as interstitial lung disease, pulmonary arterial hypertension, and digital ulcers [2]. SSc treatment requires both immune-modulating therapies for certain clinical manifestations and vascular therapies for microangiopathy. No disease-modifying drugs for SSc exist; a close follow-up with a regular screening of organ involvement in these patients appears to improve mortality [3].

The Scleroderma Unit is a national referral center for this rare connective tissue disease and also the Italian referral center for autologous stem cell transplantation in SSc. Our patients are almost $60 \%$ from the North of Italy and $40 \%$ from the rest of the country.

Our normal clinical activity is characterized by outpatient visits, videocapillaroscopy exam, ulcers treatment (including digital lipofilling), therapeutic infusions in day hospital regimen (prostanoids, intravenous biologic drugs, and immunoglobulin), and clinical trials. Four physicians, four nurses, two of whom specialized in ulcers advanced medications, and one data manager for clinical trials are involved.

\section{How to take care of SSc patients in the COVID-19 era?}

Great importance in the mortality associated with COVID-19 has been given to the concomitant comorbidities (diabetes, hypertension, cardiovascular disease, lung disease, chronic liver disease, and malignancies) of the patients, but no extensive case series of COVID-19 in SSc patients are reported in literature to date [4]. Since lung diseases and immunosuppression are WHO-defined risk factors for a more severe course of COVID-19, SSc patients could represent a subgroup of patients at increased risk of respiratory or life-threatening complications.
After COVID-19 outbreak and consequent regional and national legislations, only urgent visits and therapeutic treatments have been admitted in the Italian hospitals, including the Gaetano Pini hospital. Then, we had to reorganize our Scleroderma Unit in order to manage adequately all SSc patients, balancing the need to cure and the need to reduce the risk of contagion.

\section{Education}

A good education of patients with SSc during the SARS-CoV2 outbreak was mandatory. Each patient has been invited to follow WHO and Italian Ministry of Health recommendations: social distancing; frequent hand wash; use of masks and gloves; and avoid touching nose, eyes and mouth.

We also gave patient answers and support regarding working issues, especially for SSc patients working in settings of high-risk infection.

\section{Home therapy management}

All patients have been invited to maintain home therapies, even the immunosuppressive treatments, unless the appearance of symptoms was suggestive of an infectious disease. The risk of dangerous disease flares after suspension of immunosuppressive treatment is high and should be avoided. The Italian Society of Rheumatology (SIR) provided a reply to frequently asked questions (FAQ) among rheumatic patients facing up COVID-19 that our Unit shared with SSc patients (https://www.reumatologia.it/cmsx.asp?IDPg=1087).

\section{Keep in contact}

Using different ways of communication (phone call, email, sharing of photos and files), we have assured an easy and constant contact with SSc patients unable to reach the Hospital, guiding them in the management of the disease, from a clinical, therapeutic, and bureaucratic point of view.

The therapeutic continuity has been guaranteed through the evaluation of laboratory and/or instrumental tests and symptoms reported by the patients.

During these contacts, we also have assessed the risk of COVID-19 in SSc patients, investigating for the presence of fever, cough, dyspnea, sore throat, rhinorrhea, fatigue, anosmia, and all the other typical symptoms of COVID-19 and asking about known contacts with COVID-19 patients.

In Italy, dedicated numbers for sanitary assistance of COVID-19 cases have been established; therefore, in the presence of symptoms indicative of moderate to severe COVID19 (dyspnea, desaturation), the patients have been invited to call for assistance. 


\section{Hospital reorganization}

Since the first epidemic appearance in Italy, checkpoints have been set up at hospitals and department entrances. A nurse asks for symptoms suggestive of COVID-19 in the last 15 days, measures body temperature, and provides hand sanitizer and surgical mask to the patients and personal staff, reporting on a register names, dates, and time of entry. The access is forbidden for caregivers, and outpatient visits are scheduled in order to avoid overcrowding in waiting rooms. Doctors, nurses, and all the hospital staff wear the proper personal protective equipment (PPE) and follow the recommendations to prevent contamination.

Day hospital infusions are organized in respect of the distance needed between patients (at least $2 \mathrm{~m}$ ). SSc patients living faraway from Lombardy who need infusion therapies have been referred to their closest hospital, temporarily.

\section{Ulcer medications}

The service of ulcers and wound medication has been maintained for severe cases. SSc patients with ulcers who cannot reach the Hospital, due to the lockdown, have been followed as well, since our specialized nurses guided caregivers or home nurse on the correct management, with the aid of video calls and photos.

\section{Hospital drug dispensation}

Our Pharmacy, in accordance with territorial sanitary agencies or pharmaceuticals companies, has organized home deliveries of drugs only supplied by hospital pharmacies in Italy, such as mycophenolate mofetil, macitentan, selexipag, and subcutaneous biologics.

\section{Conclusion}

The SARS-Cov-2 outbreak has led to a rapid reorganization of Italian Public Health system to assure COVID-19 patients assistance, with a conversion of a large number of hospitals and clinics in COVID-19-specialized centers. Clinical management of patients with chronic diseases included rheumatologic patients, which risk to be sacrificed in order to respond to COVID-19 pressure. The organization of our Scleroderma Unit has been quickly rethought to assure SSc patients assistance in safety for them and health-care workers. The use of electronic way of communication and a frequent virtual contact with SSc patient have allowed a continuity of care also outside the Hospital.

Authors' contributions A.M. and N.D.P. have planned and written the manuscript. W.M., F.P., C.C., and R.C. made comments. All authors approved the final submission.

Funding information This research did not receive any specific grant from funding agencies in the public, commercial, or not-for-profit sectors.

Data availability Not applicable.

\section{Compliance with ethical standards}

Disclosures None.

Code availability Not applicable.

\section{References}

1. Deng SQ, Peng HJ (2020) Characteristics of and public health responses to the coronavirus disease 2019 outbreak in China. J Clin Med 9(2):E575. https://doi.org/10.3390/jcm9020575

2. Gabrielli A, Avvedimento EV, Krieg T (2009) Scleroderma. N Engl J Med 360(19):1989-2003. https://doi.org/10.1056/ NEJMra0806188

3. Denton CP, Khanna D (2017) Systemic sclerosis. Lancet. 390(10103):1685-1699. https://doi.org/10.1016/S0140-6736(17) 30933-9

4. Rodriguez-Morales AJ, Cardona-Ospina JA, Gutiérrez-Ocampo E et al (2020) Latin American Network of Coronavirus Disease 2019-COVID-19 Research (LANCOVID-19). Clinical, laboratory and imaging features of COVID-19: a systematic review and metaanalysis. Travel Med Infect Dis:101623 in press. https://doi.org/10. 1016/j.tmaid.2020.101623

Publisher's note Springer Nature remains neutral with regard to jurisdictional claims in published maps and institutional affiliations. 